\title{
DECONVOLUTION OF 3D FLUORESCENCE MICROGRAPHS WITH AUTOMATIC RISK MINIMIZATION
}

\author{
Sathish Ramani, Cédric Vonesch and Michael Unser \\ Biomedical Imaging Group, \\ Ecole Polytechnique Fédérale de Lausanne (EPFL), \\ CH-1015 Lausanne, Switzerland.
}

\begin{abstract}
We investigate the problem of automatic tuning of a deconvolution algorithm for three-dimensional (3D) fluorescence microscopy; specifically, the selection of the regularization parameter $\lambda$. For this, we consider a realistic noise model for data obtained from a CCD detector: Poisson photon-counting noise plus Gaussian read-out noise. Based on this model, we develop a new risk measure which unbiasedly estimates the original mean-squared-error of the deconvolved signal estimate. We then show how to use this risk estimate to optimize the regularization parameter for Tikhonov-type deconvolution algorithms. We present experimental results on simulated data and numerically demonstrate the validity of the proposed risk measure. We also present results for real 3D microscopy data.
\end{abstract}

Index Terms - 3D fluorescence microscopy, Deconvolution, Unbiased risk estimate (URE), CCD Noise.

\section{INTRODUCTION}

Deconvolution is widely used to enhance three-dimensional (3D) fluorescence microscopy images, both to facilitate their visual inspection and to improve the results of subsequent computer-assisted analysis steps [1]. Although many deconvolution techniques are available [2], they seldom yield agreeable results without human intervention-they are not completely automatic. In this context, an oft encountered problem is the selection of the appropriate regularization parameter $\lambda$. Some well-known techniques that address this issue are the generalized cross validation (GCV) [3] and L-curve based methods. But these are mostly empirical and do not directly minimize the mean-squared-error (MSE) of the signal estimate which is the most frequently used measure to quantify algorithm performance. However, since the MSE depends on the noise-free signal it cannot be used for real data. A practical approach, therefore, is to replace the MSE by an unbiased estimate (commonly termed as the unbiased risk estimateURE) that is computed entirely from the noisy data and the deconvolved signal estimate. Even though this concept is well-known in the statistics literature and has been used in denoising applications, it has not received considerable attention

This work was supported by Centre d'Imagerie BioMédicale (CIBM), the Hasler Foundation, and the Swiss National Science Foundation (SNSF grant 200020-109415). The authors thank Prof. Pierre Gönczy, EPFL/EDBC-GE, for kindly providing the sample that was used to produce the micrographs. for deconvolution problems. Specifically, UREs are available for the individual cases of additive Gaussian noise (Stein's unbiased risk estimate-SURE [4]) and signal-dependent Poisson noise [5], respectively. However, measurement noise due to a CCD detector is never entirely Poisson nor additive Gaussian: a realistic model is to consider a mixture of both [6].

Our first contribution in this paper is a new URE that takes into account Poissonian photon-counting noise and Gaussian read-out noise. To the best of our knowledge, this has neither been documented before, nor applied for deconvolution of 3D fluorescence microscopy images. In this work, we therefore propose to select $\lambda$ by minimizing this URE. The computation of the proposed URE requires the knowledge of the gain of the Poisson component and the mean and the variance of Gaussian read-out noise. These quantities depend on the calibration of the microscope and are either unspecified or difficult to measure in practice. We therefore also propose a simple technique to estimate these parameters from the given data which is our second contribution. Thus, the new URE combined with estimated CCD parameters provide a completely data-driven mechanism of optimizing $\lambda$ for regularized deconvolution.

In what follows, we briefly describe the image formation model and give the formula for the new URE that corresponds to this model. We then show how to compute this for Tikhonov deconvolution and validate it experimentally by presenting numerical results on simulated data. Following that we apply our method to real 3D microscopy data.

\section{IMAGE FORMATION MODEL}

The image formation of a wide-field microscope is classically described by the following 3D convolution model [1]:

$$
Y(x, y, z)=(h * \chi)(x, y, z),
$$

where $\chi(x, y, z)$ is the $3 \mathrm{D}$ object of interest (we have neglected the effect of the background), $Y(x, y, z)$ is the intensity map of the blurred object and $h(x, y, z)$ (under the paraxial approximation) is the shift-invariant blur function of the microscope [1]. Typically, the blurred image $Y$ is digitized by a CCD detector array which, in the process, introduces measurement noise. There are many sources of noise (such as photon shot noise, background noise, dark current, read-out noise and other deterministic noises) that arise in CCD detectors $[1,6]$. However, for numerical tractability, we restrict ourselves to the following principal components: 
the Poisson photon-counting noise and the Gaussian read-out noise, which adequately describe the CCD characteristics [7]. Moreover, we apply a suitable discretization of the continuous convolution in (1) which leads to the following matrix formulation of the problem:

$$
\mathbf{y}=\alpha \mathcal{P}\{\mathbf{H} \mathbf{x}\}+\mathbf{b},
$$

where $\alpha$ is the gain factor and the operator $\mathcal{P}\{\cdot\}$ represents the effect of Poisson noise; the mean and variance of the Gaussian read-out noise $\mathbf{b}$ are denoted by $m$ and $\sigma^{2}$, respectively and the matrix $\mathbf{H}$, which approximates the continuously defined $h$, is treated as block-circulant. Additionally, we assume independence of the individual components of $\mathbf{y}$ and that of the photon-counting process and the read-out noise.

\section{PROBLEM STATEMENT}

Given $\mathbf{y}$, we are interested in obtaining an estimate $\tilde{\mathbf{x}}$ of the noise-free signal $\mathbf{x}$. This is performed by the application of a suitably regularized deconvolution algorithm [2] which may be represented by the mapping $\mathbf{f}_{\lambda}: \mathbb{R}^{N} \rightarrow \mathbb{R}^{N}$ that operates on $\mathbf{y}$ to yield $\tilde{\mathbf{x}}$, that is, $\tilde{\mathbf{x}}=\mathbf{f}_{\lambda}(\mathbf{y})$, where $\lambda$ is the regularization parameter. As discussed earlier, in this work, we focus on obtaining the "best" signal estimate in the MSE sense, that is, choose $\lambda$ so that $\mathbf{f}_{\lambda}(\mathbf{y})$ minimizes the MSE given by

$$
\operatorname{MSE}\left(\mathbf{f}_{\lambda}(\mathbf{y})\right)=\frac{1}{N}\left\|\mathbf{x}-\mathbf{f}_{\lambda}(\mathbf{y})\right\|^{2} .
$$

To get around the dependency of the MSE on the noise-free signal $\mathbf{x}$, we develop a new practicable risk measure that unbiasedly estimates $\operatorname{MSE}\left(\mathbf{f}_{\lambda}(\mathbf{y})\right)$ for the data model described by (2).

\subsection{Unbiased Risk Estimate-URE}

In what follows, we will assume that $\mathbf{f}_{\lambda}$ is a bounded and continuous operator (continuous in the input data) and that its divergence w.r.t $\mathbf{y}$ given by $\operatorname{div}_{\mathbf{y}}\left\{\mathbf{f}_{\lambda}(\mathbf{y})\right\}=\sum_{n=1}^{N} \frac{\partial \mathrm{f}_{\lambda n}(\mathbf{y})}{\partial y_{n}}$, where $y_{n}$ and $\mathrm{f}_{\lambda n}(\mathbf{y})$ are the $n^{\text {th }}$ component of the vectors $\mathbf{y}$ and $\mathbf{f}_{\lambda}(\mathbf{y})$, respectively, is well-defined in the weak sense. Then, for $\mathbf{y}$ in (2), we define the risk estimate as the random variable

$$
\begin{aligned}
\eta\left(\mathbf{f}_{\lambda}(\mathbf{y})\right)= & \frac{1}{N}\|\mathbf{x}\|^{2}+\frac{1}{N}\left\|\mathbf{f}_{\lambda}(\mathbf{y})\right\|^{2}+\frac{2 \sigma^{2}}{N \alpha} \operatorname{div}_{\mathbf{y}}\left\{\overline{\mathbf{g}}_{\lambda}(\mathbf{y})\right\} \\
& -\frac{2}{N \alpha}(\mathbf{y}-m \mathbf{1})^{\mathrm{T}} \overline{\mathbf{g}}_{\lambda}(\mathbf{y})
\end{aligned}
$$

where 1 is a $N \times 1$ column vector of 1 's, $\overline{\mathrm{g}}_{\lambda n}(\mathbf{y})=\overline{\mathrm{f}}_{\lambda n}(\mathbf{y}-$ $\left.\alpha \mathbf{e}_{n}\right), \mathbf{f}_{\lambda}(\mathbf{y})=\mathbf{H}^{\mathrm{T}} \overline{\mathbf{f}}_{\lambda}(\mathbf{y})$ and $\mathbf{e}_{n}$ is a $N \times 1$ vector whose components are all zero except for the $n^{\text {th }}$ one which is unity. The following theorem ensures that $\eta\left(\mathbf{f}_{\lambda}(\mathbf{y})\right)$ is indeed unbiased.

Theorem 1 The random variable $\eta\left(\mathbf{f}_{\lambda}(\mathbf{y})\right)$ is an unbiased estimate of $\operatorname{MSE}\left(\mathbf{f}_{\lambda}(\mathbf{y})\right)$, that is,

$$
E_{\mathbf{y}}\left\{\eta\left(\mathbf{f}_{\lambda}(\mathbf{y})\right)\right\}=E_{\mathbf{y}}\left\{\operatorname{MSE}\left(\mathbf{f}_{\lambda}(\mathbf{y})\right\},\right.
$$

where $E_{\mathbf{y}}\{\cdot\}$ is the expectation operator w.r.t $\mathbf{y}$.
The proof of this result will appear elsewhere. The unbiasedness indicates the equality of $\eta$ and MSE in the event $N \rightarrow \infty$, where $N$ is the number of pixels. For practical purposes, therefore, $\eta$ can be used as a reliable substitute for MSE for very large $N$ (especially image stacks). Moreover, we note that, except for the first term $\|\mathbf{x}\|^{2}$ in $\eta$ (which is a non-relevant constant for our purpose), all the other terms are purely derived from $y$ and therefore computable. In this work, we propose to minimize $\eta\left(\mathbf{f}_{\lambda}(\mathbf{y})\right)$ for finding the optimal $\lambda$.

\section{DECONVOLUTION ALGORITHM}

The URE $\eta$ has a closed form expression that is in principle computable for certain classes of estimators. In the sequel, we restrict ourselves to the linear setting which is adequate for our purpose and completely tractable mathematically. We consider the Tikhonov-regularized algorithm [2] which yields an explicit expression for $\mathbf{f}_{\lambda}$. The signal estimate is obtained by minimizing the Tikhonov criterion $\mathcal{J}_{\text {Tik }}$ :

$$
\begin{aligned}
\mathbf{f}_{\lambda}(\mathbf{y}) & =\arg \min _{\mathbf{u}} \mathcal{J}_{\operatorname{Tik}}\{\mathbf{u}\} \\
\mathcal{J}_{\operatorname{Tik}}\{\mathbf{u}\} & =\|\mathbf{y}-\mathbf{H u}\|^{2}+\lambda\|\mathbf{L u}\|^{2},
\end{aligned}
$$

where $\mathbf{L}$ is the matrix (typically block-circulant) that represents a suitable regularization operator (laplacian or gradient) and $\lambda$ is a positive scalar that controls the degree of regularization applied to the solution. The solution of (6) takes the classical form

$$
\mathbf{f}_{\lambda}(\mathbf{y})=\underbrace{\left(\mathbf{H}^{\mathrm{T}} \mathbf{H}+\lambda \mathbf{L}^{\mathrm{T}} \mathbf{L}\right)^{-1}}_{\mathbf{A}_{\lambda}} \mathbf{H}^{\mathrm{T}} \mathbf{y} .
$$

When $\mathbf{H}$ and $\mathbf{L}$ are block-circulant, so is $\mathbf{A}_{\lambda}$. Then the above matrix solution is efficiently implemented via FFTs. Moreover, commutativity of $\mathbf{A}_{\lambda}$ and $\mathbf{H}^{\mathrm{T}}$ (circulant matrices) yields $\overline{\mathbf{f}}_{\lambda}(\mathbf{y})=\mathbf{A}_{\lambda} \mathbf{y}$. Thus the URE (without the constant $\|\mathbf{x}\|^{2}$ ) can be deduced as

$$
\begin{aligned}
\eta_{\mathrm{Tik}}\left(\mathbf{f}_{\lambda}(\mathbf{y})\right)= & \frac{1}{N}\left\|\mathbf{f}_{\lambda}(\mathbf{y})\right\|^{2}+ \\
& \frac{2}{N \alpha}\left(\begin{array}{l}
\left(\sigma^{2}-m \alpha\right) \operatorname{Trace}\left\{\mathbf{A}_{\lambda}\right\} \\
-(\mathbf{y}-m \mathbf{1})^{\mathrm{T}} \mathbf{A}_{\lambda} \mathbf{y} \\
+\alpha \operatorname{Trace}\left\{\mathbf{D}_{\mathbf{y}} \mathbf{A}_{\lambda}\right\}
\end{array}\right),
\end{aligned}
$$

where $\mathbf{D}_{\mathbf{y}}=\operatorname{diag}\{\mathbf{y}\}$. The circulant nature of $\mathbf{A}_{\lambda}$ facilitates the computation of Trace $\left\{\mathbf{A}_{\lambda}\right\}$ and Trace $\left\{\mathbf{D}_{\mathbf{y}} \mathbf{A}_{\lambda}\right\}$. The former is easily computed in the Fourier domain: it is the sum of the corresponding DFT coefficients, while the latter simplifies to $\operatorname{Trace}\left\{\mathbf{D}_{\mathbf{y}} \mathbf{A}_{\lambda}\right\}=\frac{1}{N} \operatorname{Trace}\left\{\mathbf{A}_{\lambda}\right\} \mathbf{1}^{\mathrm{T}} \mathbf{y}$.

\section{ESTIMATION OF CCD PARAMETERS}

The use of $\eta_{\text {Tik }}$ requires the knowledge of the CCD dependent parameters $\left(\alpha, m, \sigma^{2}\right)$. While $m$ and $\sigma^{2}$ (of the read-out noise) may be measured by running separate calibration experiments [6], $\alpha$ is not totally synonymous with the gain that is typically provided in microscopes and must therefore be estimated from the given data. For this purpose, we develop a simple procedure that is essentially based on the following 


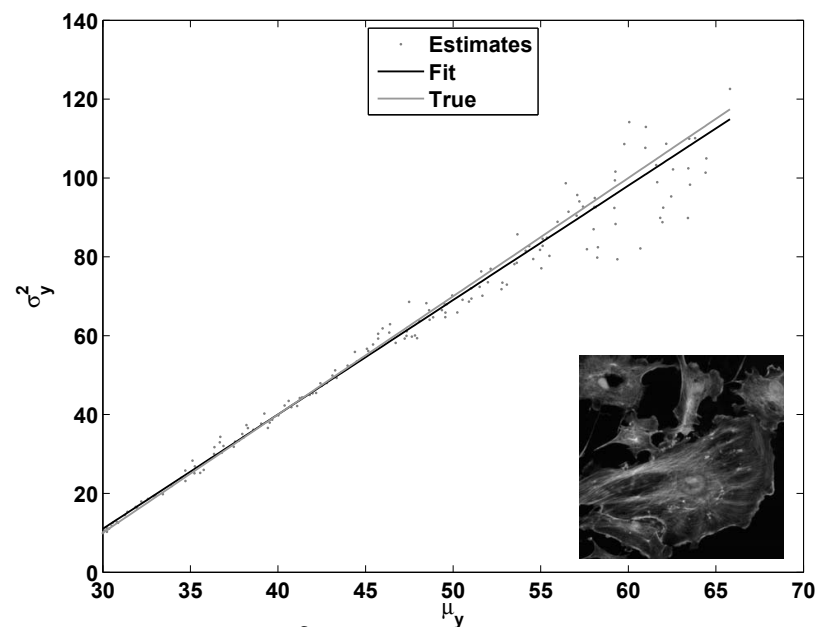

Fig. 1. Plot of $\left(\hat{\mu}_{\mathbf{y}}, \hat{\sigma}_{\mathbf{y}}^{2}\right)$ and corresponding fit for a noisy realization $\left(\alpha, m, \sigma^{2}\right)=(3,30,10)$ of the image shown in the inset. Estimated parameters are $(2.91,29.65,9.99)$.

identities:

$$
\begin{aligned}
\mu_{\mathbf{y}}[k] \stackrel{\text { def }}{=} E_{\mathbf{y}}\left\{y_{k}\right\}=\alpha(\mathbf{H x})_{k}+m, \\
\sigma_{\mathbf{y}}^{2}[k] \stackrel{\text { def }}{=} \operatorname{Var}_{\mathbf{y}}\left\{y_{k}\right\}=\alpha^{2}(\mathbf{H x})_{k}+\sigma^{2},
\end{aligned}
$$

where $(\mathbf{H x})_{k}$ is the $k^{\text {th }}$ component of $\mathbf{H x}$. This leads to the relationship $\sigma_{\mathbf{y}}^{2}[k]=\alpha \mu_{\mathbf{y}}[k]+\left(\sigma^{2}-m \alpha\right)$. Then the idea is to estimate $\mu_{\mathbf{y}}[k]$ and $\sigma_{\mathbf{y}}^{2}[k], k=1,2, \ldots, K$ and perform a linear regression analysis on $\left(\mu_{\mathbf{y}}[k], \sigma_{\mathbf{y}}^{2}[k]\right)_{k=1}^{K}$ : the slope yields the gain $\alpha$ and the intercept yields the constant $\kappa=$ $\left(\sigma^{2}-m \alpha\right)$.

To estimate $\left(\mu_{\mathbf{y}}[k], \sigma_{\mathbf{y}}^{2}[k]\right)_{k=1}^{K}$, we first segment $\mathbf{y}$ into $K$ non-overlapping regions wherein the underlying blurred signal $\mathbf{H x}$ is approximately constant in each region. This is accomplished by running a very few number of iterations (typically $<4$ ) of heavily regularized 2D total variation denoising (TVD) on each slice of $\mathbf{y}$ - the advantage of using TVD is that it not only reduces the noise in $\mathbf{y}$ but automatically yields a piecewise constant approximation $\mathbf{y}_{\mathrm{PC}}$ of $\mathbf{H x}$. We then define level values $\epsilon_{k}=\min \left\{\mathbf{y}_{\mathrm{PC}}\right\}+(k-1) \delta$ and corresponding segments $\Omega_{k}=\left\{1 \leq i \leq N: \epsilon_{k}-\frac{\delta}{2} \leq y_{\mathrm{PC} i}<\epsilon_{k}+\frac{\delta}{2}\right\}, k=$ $1,2, \ldots, K$ and $\delta$ is a predefined width of each level. This basically ensures a non-redundant partitioning of the image $\mathbf{y}$ : $\Omega_{k} \bigcap \Omega_{l}=\emptyset, k \neq l$ and $\bigcup_{k=1}^{K} \Omega_{k}=\mathcal{I}_{N}$, where $\mathcal{I}_{N}=$ $\{1,2, \ldots, N\}$.

Estimates of $\mu_{\mathbf{y}}[k]$ and $\sigma_{\mathbf{y}}^{2}[k]$ are computed for each segment as the corresponding sample mean and sample variance, respectively: $\hat{\mu}_{\mathbf{y}}[k]=\frac{1}{N_{k}} \sum_{i \in \Omega_{k}} y_{i}, \hat{\sigma}_{\mathbf{y}}^{2}[k]=\frac{1}{N_{k}-1} \sum_{i \in \Omega_{k}}$ $\left(y_{i}-\hat{\mu}_{\mathbf{y}}[k]\right)^{2}$, where $N_{k}$ is the cardinality of the set $\Omega_{k}$. Since most of the noise is sufficiently reduced in $\mathbf{y}_{\mathrm{PC}}$, assuming that $\min \{\mathbf{H} \mathbf{x}\}=0$ (which is mostly the case with biological images), $m$ is simply estimated as $\min \left\{\mathbf{y}_{\mathrm{PC}}\right\}$. Thus, knowing $\alpha, \kappa$ and $m$, we easily obtain a formula for estimating $\sigma^{2}$.

\section{EXPERIMENTS}

First, we numerically validate the proposed URE (9) and the estimation scheme described in Section 5. For this, we per-

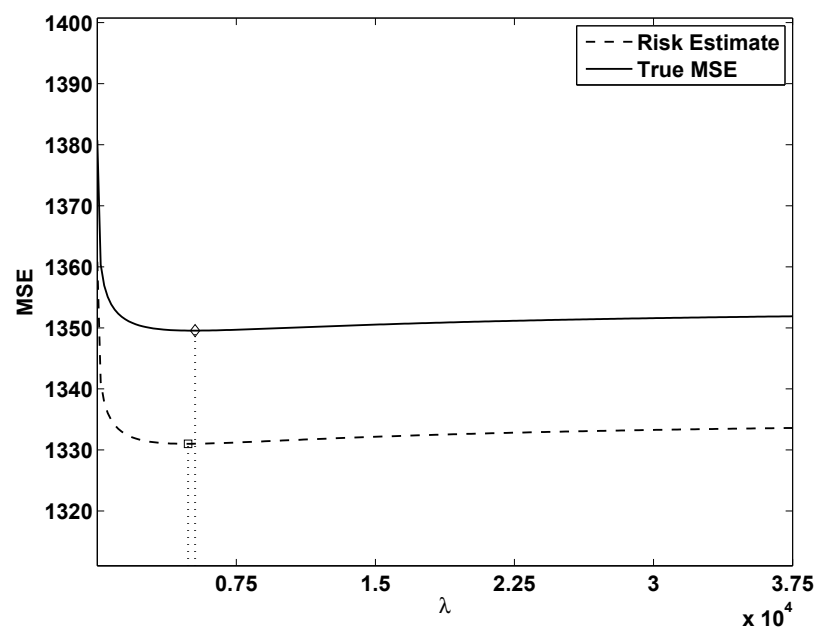

Fig. 2. Plot of the MSE and corresponding estimate (both without the $\|\mathbf{x}\|^{2}$ term) as functions of $\lambda$. The minimum of both quantities are indicated by corresponding markers.

formed the following experiment: we used the 2D uniform blur kernel of size $9 \times 9$ and generated a noisy realization of the image shown in the inset of Figure 1 according to (2) for the following parameter setting: $\left(\alpha, m, \sigma^{2}\right)=(3,30,10)$, $\mathrm{BSNR} \approx 6.77 \mathrm{~dB}$. From the noisy image, we estimated the CCD parameters using the method described in Section 5 with $\delta=0.2$. Figure 1 shows the corresponding regression plot. The estimated parameter values are $\hat{\alpha}=2.91, \hat{m}=29.65$, and $\hat{\sigma}^{2}=9.99$ : it is clearly seen that the fit (black line) almost overlaps with the true relationship (gray line) between $\mu_{\mathbf{y}}$ and $\sigma_{\mathbf{y}}^{2}$.

We then applied the deconvolution algorithm (8) using a Laplacian operator for $\mathbf{L}$. The URE $\eta_{\text {Tik }}$ was computed using the estimated CCD parameter values and plotted in Figure 2 in comparison with the MSE (equation (3) without the $\|\mathbf{x}\|^{2}$ term). It is clearly seen that the URE correctly predicts the shape of MSE curve for the entire range of $\lambda$. The slight offset $(\approx 20)$ is due to the multiplicative nature of the gain $\alpha$ : even a slight difference from the true value drastically shifts the URE vertically. But this does not move the optimal value of $\lambda$, as seen in Figure 2 where the URE closely determines the minimum of the MSE. For comparison, we also tested the modified GCV of [3] for Tikhonov regularization and found that it always yielded a $\lambda$ that was at least an order of magnitude away from the optimal one. The lesser performance of GCV may be due to the fact that this measure is linked to the MSE in the measurement domain which is suitable for denoising, but less appropriate for deconvolution.

We now present results for automatic Tikhonov deconvolution of real 3D wide-field fluorescence micrographs. The data was acquired on a Leica DM 5500B system equipped with a cooled CCD camera, at the EPFL BioImaging and Optics Platform. Figure 4 (top) shows the maximum-intensity projection (MIP) of the original 3D image stack of a C. Elegans embryo $(464 \times 320 \times 270$ voxels $)$. The sample was labeled with two fluorophores: Alexa488 (microtubules, green channel) and Alexa568 (vesicles, red channel). The blurring matrix $\mathbf{H}$ for the deconvolution algorithm was generated 


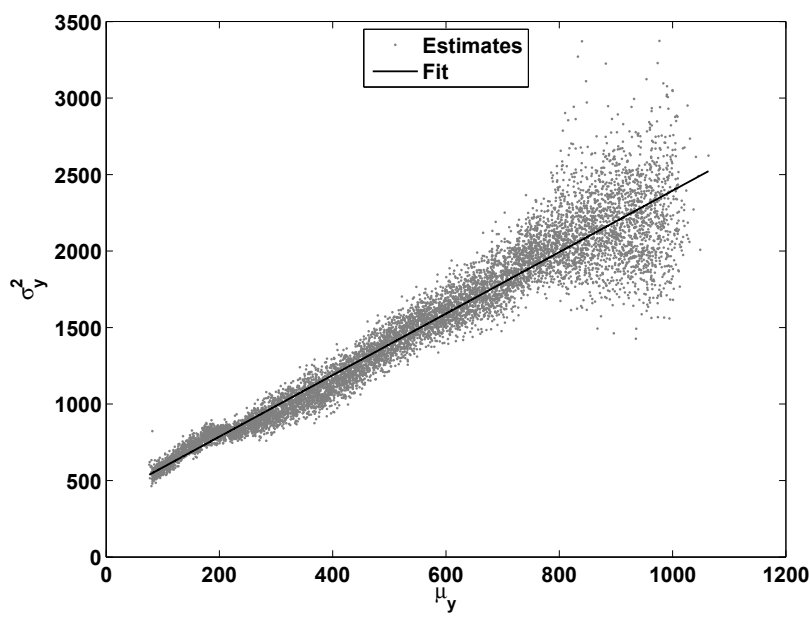

Fig. 3. Plot of $\left(\hat{\mu}_{\mathbf{y}}, \hat{\sigma}_{\mathbf{y}}^{2}\right)$ and corresponding fit $\left(\hat{\alpha}, \hat{m}, \hat{\sigma}^{2}\right)=$ $(2.01,84.72,555.07)$, for the green channel of the noisy stack shown in Figure 4 (top).

mathematically based on a standard diffraction-limited PSF model [1] using the manufacturer-provided imaging parameters (magnification: $63 \times$; numerical aperture: 1.4 ; immersion oil refractive index: 1.518 ).

Figure 3 documents the linear regression on $\left(\mu_{\mathbf{y}}, \sigma_{\mathbf{y}}^{2}\right)$ estimated for data in the green channel. For this data set, MATLAB (FFT-based) implementation of the Tikhonov deconvolution algorithm required approximately 3.6 GB of memory and took about 30 seconds to produce a deconvolved signal estimate for a single $\lambda$ on a $2.66 \mathrm{GHz}$ (dual core) Intel-Mac with 6 GB RAM. The two channels were processed separately using the method described above for $\lambda$ that minimized $\eta_{\text {Tik }}$. The MIP of the deconvolved data set is shown in Figure 4 (bottom). It is seen that the typical wide-field haze is greatly reduced. In particular, the red light coming from the vesicles has been reassigned to a more concentrated and brighter volume; this is in accordance with the fact that vesicles are relatively small biological structures. Also, the filament structure of the microtubules is visible with significantly better contrast.

\section{CONCLUSION}

In this work, we considered the problem of data-driven selection of the optimal regularization parameter $\lambda$ (in the minimum MSE sense) for regularized deconvolution of 3D fluorescence microscopy. For this, we developed a new unbiased risk estimate (URE) that takes into account Poisson photoncounting noise and Gaussian read-out noise and proposed to choose $\lambda$ by minimizing this measure. We also devised a scheme for estimating the CCD parameters (the detector gain, mean and variance of the read-out noise) necessary for computing the proposed URE from the given data. We validated our method using phantom data and presented deconvolution results for real 3D wide-field microscopy images.

\section{REFERENCES}

[1] C. Vonesch, F. Aguet, J. -L. Vonesch, and M. Unser, "The colored revolution of bioimaging," IEEE Signal Process.
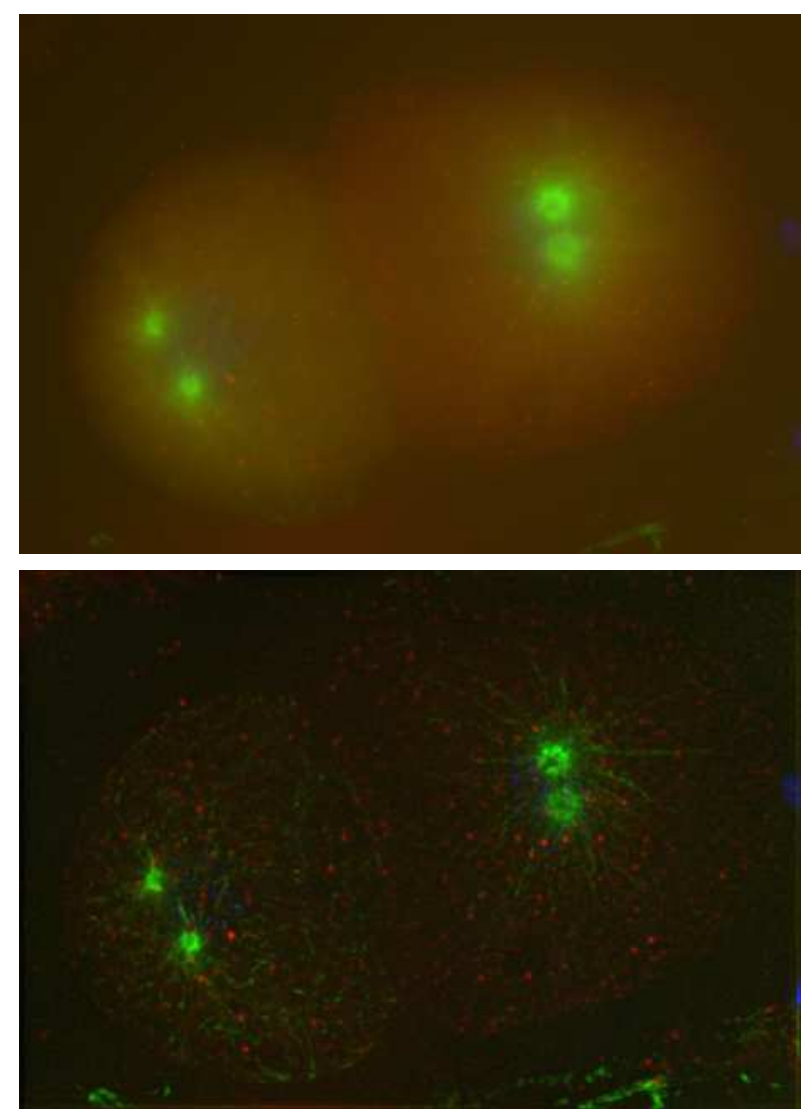

Fig. 4. Maximum intensity projections of acquired (top) and deconvolved (bottom) image stack of a C. Elegans embryo.

Mag., vol. 23, no. 3, pp. 20-31, 2006.

[2] P. Sarder and A. Nehorai, "Deconvolution methods for 3-D fluorescence microscopy images," IEEE Signal Process. Mag., vol. 23, no. 3, pp. 32-45, 2006.

[3] L. H. Schaefer, D. Schuster, and H. Herz, "Generalized approach for accelerated maximum likelihood based image restoration applied to three-dimensional fluorescence microscopy," J. Microsc.-Oxford, vol. 204, Part 2, pp. 99-107, November 2001.

[4] C. Stein, "Estimation of the mean of a multivariate normal distribution," Ann. Statist., vol. 9, pp. 1135-1151, 1981 .

[5] H. M. Hudson, "A natural identity for exponential families with applications in multiparameter estimation," Ann. Statist., vol. 6, no. 3, pp. 473-484, 1978.

[6] D. L. Snyder and A. M. Hammoud, "Image recovery from data acquired with a charge-coupled-device camera," $J$. Opt. Soc. Am. A, vol. 10, no. 5, pp. 1014-1023, 1993.

[7] H. Lanteri and C. Theys, Inverse Problems and Image deconvolution, vol. 22, pp. 3-33, EAS Publications, 2006. 